HStud 26 (2012)2, 285-302

DOI: 10.1556/HStud.26.2012.2.8

\title{
DER BRIEF ALS DIE APPELLSTRUKTUR DER GESTALTUNG IN KEMÉNY ZSIGMONDS ROMAN GYULAI PÁL
}

\author{
BORBÁLA PINTÉR \\ Eötvös Loránd Universität \\ Budapest, Ungarn
}

\begin{abstract}
Die neue Tendenzen suchende Kemény-Rezeption ist bis zu einer bedeutenden rezeptionsgeschichtlichen Tradition geworden. Vorliegende Arbeit schließt sich der Tradition des Wiederlesens von Keménys Werk an und beschäftigt sich mit Zsigmond Keménys Roman Gyulai Pál. Gyulai Pál als Experiment schöpft aus einem großen Formenrepertoir, strebt auf den Normen der Romantik beruhend nach der Veränderung der Funktionen der Gattungselemente. Während der Roman einerseits erst die sprachlich-poetischen Normen der einzelnen Gattungskonventionen erschafft, stellt er das Artistische des Verfahrens in den Vordergrund. Von den verschiedenen Gattungskonstruktionen konzentriert sich dieVerfasserin dieser Studie auf die in dem Roman integrierten Briefe, die als strukturelle Einlagen interpretiert werden, sich in der Fiktion in den Haupttext einbauen, die ineinanderknüpfende Dynamik der diskursiven und narrativen Schichten unterbrechen. In dem Fall der Briefeinlagen wird ein eingebetteter Textkörper angenommen. Die Ordnung der herkömmlichen Diegese wird aufgehoben, um den Leser über Ereignisse und Charakterzüge zu informieren, für deren Bekanntgabe die bisherige Erzählweise sich nicht als geeignet erwies. Der Wechsel der Sprechsituationen und der Gattungskonventionen, das Drama, den Epos, den Brief und das Tagebuch in Einheit verschmelzende Form schafft eine Metanarrativik, die die Konstruiertheit des Textes betont, und von dem jeweiligen Leser erwartet, sich in dieser konzeptionell geschaffenen Welt auszukennen. In dem romantischen Plotentwurf und der Realitätsnähe entdecken wir außertextuelle Elemente der Wirklichkeit, während im Erzählen und in der Konstruiertheit die modernen Merkmale der Textualität zur Geltung kommen.
\end{abstract}

Schlagwörter: Gestaltung, Brief, Gattungsvielfalt, Leerstellen, narrative Methode und Struktur, dynamisches Motiv der Handlungsgestaltung, Metalepse, selbstreflexive Konstruktion

Die literaturgeschichtliche Tradition hat die Kemény-Werken lange in der Dialektik der Romantik und des Realismus interpretiert. Später begann sich der disjunktive Charakter dieses Verhältnisses zu verstärken. In den letzten Jahren eröffnete die Monografie von Mihály Szegedy-Maszák ${ }^{1}$ die Möglichkeit vor einer neue Tendenzen suchenden Kemény-Rezeption - eine Bestrebung, die bis in un- 
sere Tage zur rezeptionsgeschichtlichen Tradition wurde. Die Romane von Kemény wurden glücklicherweise von der gegenwärtigen Interpretation aus der erwähnten oppositionellen Betrachtungsweise herausgehoben. ${ }^{2}$ Generell ist die moderne Lesart ein immer häufigeres Thema in der heutigen Interpretation, wodurch immer mehr Werke des 19. Jahrhunderts einen neuen Stellenwert im gattungsgeschichtlichen Kanon erhalten.

Vorliegende Arbeit beschäftigt sich mit den Briefen in Zsigmond Keménys Roman Gyulai Pál. Das sich auf die Briefe konzentrierende Verfahren verstärkt gewisse Interpretationstendenzen auch dadurch, dass es einigen Textteilen eine besondere Wichtigkeit verleiht, während andere Perspektiven in den Hintergrund gerückt werden. Letztere werden auch nicht völlig außer Acht gelassen, nur liest dieses Verfahren diesmal nicht in den von diesen bestimmten rezeptionsgeschichtlichen Traditionen. Keménys Prosa näher betrachtend ist eine konzeptionelle Gestaltung des Themenkreises ihrer Romane zu beobachten. ${ }^{3}$ In seiner Dramen und Romane vergleichenden Poetik betont er die Themenwahl und Form betreffende Freiheit des Romans der strengen Proportionalität und Ordnung des Dramas gegenüber. Seiner Ansicht nach verfügt der Roman über ein viel reicheres Formenrepertoir, seine Originalität wurzelt in der Genauigkeit der Charakterdarstellung oder der komplizierten Handlungsführung. ${ }^{4}$

Das Streben nach einer enzyklopädischen Gattung und die in Einheit organisierte Gattungsvielfalt zeigen sich am spektakulärsten in seinem ersten Roman Gyulai Pál (1847), wo das die Personen aufzählende Titelblatt, die aus zahlreichen Szenen bestehenden, mit Regieanweisungen versehenen dramatischen Teile immer wieder von Gedichten und Epen unterbrochen werden. Der Zusammenhalt der epischen Teile wird durch essayartige Erörterungen, Parabeln, erörternde Exkurse, Brief- und Tagebuchfragmente weiter zerstückelt.

Dieses Konstruktionsprinzip kann als Synthese der Gattungen, ${ }^{5}$ als Streben nach einer umfassenden Ausdrucksform, ${ }^{6}$ als romantische Mischung der Gattungen, als absichtliche Gattungsfreiheit ${ }^{7}$ oder Freizügigkeit ${ }^{8}$ sowie als Versuch, eine im Schlegelschen Sinne über den Gattungen stehende Gattung zu schaffen, ${ }^{9}$ interpretiert werden. Thomas Cooper, der amerikanische Kemény-Forscher setzt den Roman wegen seiner konzeptionell in Einheit konstruierten Gattungsvielfalt in den europäischen Kontext, und behauptet, dass der Roman eine Collage der europäischen literarischen Vorbilder schafft, die gegensätzliche Traditionen miteinander konfrontiert und die traditionellen Formen der Struktur abschafft. ${ }^{10}$ Kemény schuf in der ungarischen Literatur des 19. Jahrhunderts also eine Gattung, deren Vorläufer in der deutschen Romantik zu suchen sind.

Die verschiedenen Gattungen, das gesungene Lied, das aufgeführte Drama, die textartige Epik und in unserem Fall der geschriebene und gelesene Brief schafft verschiedene Sprechsituationen, und setzt dadurch verschiedene Pakte zwischen Leser und Autor in Kraft. Die unterschiedlichen, sprachlich-poetischen Zielset- 
zungen fordern verschiedene Gattungskonstruktionen. Mit veränderter Erwartung hört der Leser die gesungenen Lieder an, verfolgt das dramatisierte Schicksal der Schauspieler des italienischen Ensembles, blickt auf die Gestalten einer verschwundenen Zeit zurück oder verfolgt die Entstehung bzw. den Empfang eines Brieftextes.

Auch innerhalb der Epik strebt der Roman nach einer Vielfalt der Gattungen: Außer der die Formensprache der Mündlichkeit benutzenden, auf dem kollektiven Wissen basierenden Sage oder der Gleichnisartigkeit der lehrreichen Fabel findet man erklärende, analysierende Essays, persönliche Gattungen der Bekenntnishaftigkeit: den Selbstausdruck, Selbstdefinition und Selbstverständnis bezweckenden Monolog, das Tagebuch oder den Leser ansprechenden, ihn zu einem Dialog anregenden Brief.

Es ist weiterhin wichtig festzustellen, dass man sich dem Pakt des Lesers mit dem Autor auch anschließen kann, indem man das Werk als historischen Roman liest. ${ }^{11}$ Unter historischem Roman versteht Kemény - sich von den Zeitgenossen betont distanzierend - nicht die sachliche Beschreibung der Vergangenheit, vielmehr deren künstliche Neugestaltung durch Fiktion und Vorstellungskraft. ${ }^{12}$ Das Lesen eines historischen Romans bringt unvermeidlich die Frage der Fiktionalität und Konstruiertheit ins Spiel, da die Themen- und Figurenwahl den Leser ständig an die Realität vorangegangener Texte erinnert, wobei es uns nicht vergessen lässt, dass die einzelnen historischen Figuren und Ereignisse in dem Kunstwerk in dem neuen Kontext ihre ursprüngliche Funktion verlieren, eine neue ästhetische Qualität erhalten und zum Bestandteil einer künstlerisch gestalteten Welt werden. ${ }^{13}$

Gyulai Pál als Experiment schöpft aus einem großen Formenrepertoir, strebt auf den Normen der Romantik beruhend nach der Veränderung der Funktionen der Gattungselemente. Während der Roman einerseits erst die sprachlich-poetischen Normen der einzelnen Gattungskonventionen erschafft, stellt er das Artistische des Verfahrens in den Vordergrund. Entlang dieser Gattungsgrenzen entstehen Leerstellen, durch die der Roman funktioniert; die Kombination der Textschemata wird der jeweiligen Leserfantasie überlassen und es wird dadurch für die Vielfalt der Kombinierbarkeit der Schemata gesorgt. ${ }^{14}$

Die oben dargestellte Gattungsvielfalt hat einen automatischen Einfluss auf die narrativen Methoden, auf die Behandlung der Sprechsituationen und Perspektiven und zeigt deren Komplexität. Die Sonderstellung des Erzählers und die Komplexität der Geschichtsführung lassen sich nicht bezweifeln. ${ }^{15}$ Charakteristisch sind eine äußerst starke erzählerische Anwesenheit, ${ }^{16}$ das selbst reflektierende Bewusstsein des Erzählers, ${ }^{17}$ die Vielfalt der die Abschaffung der diegetischen Ordnung bezweckenden Methoden. Die Auffädelung der Geschichten, ${ }^{18}$ die zeitlichen Sprünge, das System der Vor- und Rückblenden, ${ }^{19}$ die Unterbrechungen, ${ }^{20}$ das Verschweigen ${ }^{21}$ oder wortreiche Exkurse, ${ }^{22}$ die Kommentare des Erzählers zu 
den dramatischen Teilen, ${ }^{23}$ die Menge der die Erzählzeit ins Spiel bringenden, ${ }^{24}$ den Leser ansprechenden, sein außertextuelles Wissen aktivierenden Anreden des Lesers, ${ }^{25}$ die das Füllen der Leerstellen und die Aufdeckung des bis dahin Verschwiegenen bezweckenden Äußerungen ${ }^{26}$ und das diesem Zweck dienende letzte Kapitel ${ }^{27}$ zeugen alle von der digressiven Rolle des Erzählers. ${ }^{28}$ Der häufige Wechsel der Sprechsituationen und der Gattungskonventionen, das Drama, den Epos, den Brief und das Tagebuch in Einheit schmelzende Form schafft eine Metanarrativik die die Konstruiertheit des Textes betont, und von dem jeweiligen Leser erwartet, sich in dieser konzeptionell geschaffenen Welt auszukennen. ${ }^{29}$

Sich der Tradition des Wiederlesens des Kemény-Werkes anschließend konzentriert sich die Verfasserin dieser Studie unter den Details vor allem auf die Briefe, die als strukturelle Einlagen interpretiert werden, die sich in der Fiktion in den Haupttext einbauen, den prosaischen Textfluss, die ineinanderknüpfende Dynamik der diskursiven und narrativen Schichten unterbrechen. ${ }^{30}$

In dem Fall der Briefeinlagen wird ein eingebetteter Textkörper angenommen. Die Ordnung der herkömmlichen Diegese ${ }^{31}$ wird aufgehoben, um den Leser über Ereignisse und Charakterzüge zu informieren, für deren Bekanntgabe die bisherige Erzählweise sich nicht als geeignet erwies.

Der Brief spielt nicht nur in der Struktur des Romans eine ausgezeichnete Rolle, er ist auch ein bestimmendes, dynamisches Motiv der Handlungsgestaltung. ${ }^{32}$ Obwohl der Erzähler mehrmals darauf hinweist, kennen wir den Inhalt des von Sofronia an Boldizsár adressierten billet doux nicht, er lässt sich nur aus den Allusionen und zahlreichen Komplikationen erahnen. Wir kennen die Umstände seiner Entstehung, sein bewegtes Schicksal, wir können verfolgen, wie er in die Hände von Unbefugten gerät, und wie immer mehr von ihnen in das Geheimnis eingeweiht werden. Schließlich sind wir an der Szene beteiligt, in der der Brief in einem Kästchen zu seinem Absender zurückgelangt und dessen Schicksal besiegelt.

Den Weg des Briefes verfolgen wir aus Raumgründen nicht, und konzentrieren uns nur auf bestimmte Stationen der Entfaltung dieses Motivs, namentlich darauf, zu was für Konflikten der Besitz oder der Verlust dieses Schreibens führen kann. Genga nennt Pierro einen Verräter, verdächtigt ihn seine Aufgabe als Bote absichtlich nicht erfüllt zu haben. Schon fast am Ende der Versammlung der Muderrisen in der Komjáti-Burg beschuldigt István Farkas den Studenten Gergely, alias Ali csausz, beim Stehlen des Briefes seine astrologische Hilfe in Anspruch genommen, ihm aber den Brief nicht gezeigt und sein Geheimnis nicht verraten zu haben, was den Gesetzen der Muderris-Gesellschaft widerspricht. ${ }^{33}$ Der gestohlene Brief und der Makel an Sofronias Ehre führt zum Duell zwischen Genga und Gergely, das mit dem Tod des Adligen endet. Der Brief ist der eigentliche Grund, warum Zsigmond Sofronia sowohl aus seinem Bett als auch aus dem Land verbannt. 
Auch im Zusammenhang mit dem Brief wird der Akt des Spurenlesens kontextualisiert, in einer interpretativ-verkleinerten Spiegelszene, wo István Farkas Sofronias billet doux von Pierro stiehlt. István Farkas, um den Brief von Pierro zu beschaffen, gibt sich für einen Astrologen und Handleser aus und spielt vor, die besondere Sprache der Magie zu kennen, wobei er seine Informationen von Gergely erwirbt. In Pierros Handfläche sieht er zuerst das Zeichen von Venus, dann von Merkur erscheinen. Mit der Deutung des Ersteren warnt er seinen Zuhörer zu Vorsicht in Herzensangelegenheiten, im Letzteren sieht er das bewegte Schicksal des Boten. Pierro findet beide Deutungen richtig und fühlt sich als Postbote des Liebesbriefes immer unangenehmer, bis er mit schmerzhaftem Gesichtsausdruck zu seiner Tasche, in dem der Brief steckt, greift und dadurch dessen Fundort verrät.

Hermes ist aber nicht nur als Bote bekannt, er ist gleichzeitig auch der Gott der Diebe - eine Funktion, die Farkas absichtlich nicht erwähnt. Am ersten Tag seines Lebens warf Hermes ein Auge auf Apollos Kühe und stahl sie, indem er sie rückwärst führte und dabei umgekehrte Spuren hinterließ. Farkas führt also einen Akt der Dekodierung aus, in dem er den sich auf die konkrete Situation beziehenden, deiktischen Sinn verschweigt. Pierros Blickwinkel und Dekodierungstätigkeit sind begrenzt, er konzentriert sich nur auf die von dem Irreführenden beabsichtigte Dekodierung, während für den eingeweihten Leser die Situation der Dekodierung zugleich die Situation des Diebstahls bedeutet: Dieses Symbol zeigt sich für ihn in der Komplexität seiner Denotaten.

Auch die Umstände der Entstehung des Briefes sind ungewöhnlich: Durch den Brief von Boldizsár Báthory ins Leben gerufen, bietet Sofronia dem Grafen ihre Reize als Gegenleistung für die Rettung Sennos an. Dem Rat Gengas folgend, ihre Ehre und den Erfolg der zu erfüllenden Aufgabe vor Augen haltend, tönt sie ihr ursprüngliches Versprechen ab. Der Brief wird in Sofronias Handschrift aber nach den Anweisungen Gengas geschrieben, sie sind also beide die Autoren des Briefes. ${ }^{34}$ Der Brief gelangt in sieben Hände (Genga, István Farkas, Gergely, Jancsi Boros, Pál Gyulai und Fürst Zsigmond); seinen Inhalt kennen fünf Personen (Sofronia, Genga, Gyulai, Gergely und Zsigmond Báthory).

Das Außergewöhnliche an diesem Brief ist also, dass während er mehrere, verschiedene Handlungsstränge anbietet, zahlreiche Abenteuer und Konflikte generiert und Charaktere zur Handlung bewegt, erfüllt er seine ursprüngliche, ontologische Funktion nicht, da er nicht zu Boldizsár, dem eigentlichen Adressaten gelangt, der nicht einmal von seiner Existenz weiß. Das Ziel, das zur Entstehung des Briefes geführt hat, nämlich die Befreiung Sennos, taucht nicht einmal auf. Der Brief erfüllt also nicht seinen ursprünglichen Zweck, seine Kommunikationssituation wird aber durch die Eindringlinge verkompliziert. Die verschiedenen Figuren schreiben dem Brief verschiedene Bedeutungen zu: Für Cecil ist er ein Beweis von Sofronias Untreue, für Gergely die Möglichkeit der Vertiefung des Zwistes zwischen den beiden Verwandten, für Gyulai ein weiteres Flehen um 
Sennos Freilassung, für Zsigmond aus verschiedenen Perspektiven ein Verrat der Schauspielerin, da sie ihm nicht nur als Geliebte untreu wurde, sondern auch von Boldizsár, des Fürsten Nebenbuhler, die Freilassung seines Gegenspielers unterstützt.

Sofronias Brief erreicht seinen Zweck nicht, der Dialog zwischen der reizvollen Schauspielerin und dem übermütigen Boldizsár kommt nicht zustande. Ihre Pläne, Senno zu befreien, gehen nicht in Erfüllung, und der Brief kommt nach einer abenteuerlichen Rundreise in spöttischem Ton ergänzt zu ihr zurück. Zsigmond schenkt der Schauspielerin eine Kiste mit drei Fächern. In dem ersten Fach steckt Gold - höchstwahrscheinlich, um die Kosten der Reise zu decken. In dem zweiten eine vermutlich von Gergely verfasste Schrift mit dem Titel Fräulein Sofronias Reise, die detailliert über Sofronias Verlassen des Landes berichtet, während das dritte Fach den Originalbrief, mit Zsigmonds Handschrift ergänzt, birgt, in welcher Ergänzung er die ehemalige Prostituierte zum Verlassen des Landes auffordert. Sofronias Brief erreicht also den Adressaten nicht, stattdessen gelangt er in die Hände der Person, die ihn am wenigsten lesen soll, und von der er ergänzt wird, wodurch die ursprüngliche Absicht destruiert, der Absender vernichtet und als unerwünschte, zu entfernende Figur identifiziert wird, der ihr bisheriger Status und ihre Privilegien entzogen werden. ${ }^{35}$ Darüber berichtet die Schrift in dem zweiten Fach. Der Brief mit dieser Ergänzung wird zur Drohung, das Supplement setzt die subversive Kraft der Sprache in Betrieb, die so gegen seinen Absender wirkt und ihn vernichtet.

In die immer wieder unterbrochene Prosa des Romans Gyulai Pál werden sieben vollständige Briefeinlagen eingebettet, an anderen Stellen wechselt die Prosa zu dramatischen Szenen des Brieflesens, wenn Boldizsár Báthory und sein Sekretär Briefe lesen, und zu einer dramatisch dargestellten Situation, wenn Gyulai über die an ihn adressierten Briefe von Brutus, Senno, Boldizsár Báthory oder Márkházy nachsinnt.

Diese Arbeit beschäftigt sich mit zwei Briefen von Alfonso Cariglia näher, die wesentlich umfangreicher sind als die restlichen Briefe im Roman. Beide sind an den Grafen Tiefenbach geschrieben, der ein einziges Mal am Anfang des Romans erscheint, in einer dramatischen Szene, wo er als Geheimbote mit der Aufgabe des Wiederanschlusses von Siebenbürgen ankommt, um sich mit Alfonso und Lazintzky zu verschwören. Persönlich erscheint er im Roman nie wieder, sein Name taucht aber in verschiedenen Kontexten auf, seine geheimnisvolle Figur enthüllt sich nur durch seine Beziehungen zu anderen Figuren und an ihn adressierte Texte. Einerseit ist er in seinen geheimen Plänen der Komplize Alfonsos, andererseits wird er zum zweiten Ehemann Eleonoras. Dass die unerschütterliche, ihrem 
Mann treue Eleonora plötzlich als Gräfin Tiefenbach erscheint, wird im letzten Kapitel erklärt. ${ }^{36}$ Hier gibt der Erzähler bisher verschwiegene Einzelheiten bekannt, die Supplemente, die der Vervollständigung der Geschichte dienen sollen, bis zur letzten Sekunde zurückhaltend. Sie komplizieren aber die bereits bis dahin schon komplizierte Geschichte noch weiter, da die neuen Informationen aus den rückblickenden Textstellen den Leser zur Neudeutung der Geschehnisse zwingen. Es stellt sich zum Beispiel heraus, dass Senno ein entfernter Vewandter Tiefenbachs, das ausgesetzte Kind Hugos ist, das dieser während seines abenteuerlichen Lebens nicht findet, dem er aber ein gewisses Vermögen hinterlässt. Das Erbe wird von dem Baron an Sennos Witwe in der Hoffnung ausgezahlt, Erfolge bei ihr zu haben. Dadurch gelangt sie an ein Vermögen und einen legalen Namen, um ihre Pläne gegen Gyulai verwirklichen zu können. Ähnliche Verknüpfungen, sogar Verwicklungen der Handlungsstränge gehören zu den typischen Verfahren im Roman, die einerseits der Erfahrung der einzelnen Details, dem Füllen der Leerstellen dienen, andererseits wird der Leser gerade in Bezug auf die Kohärenz des Ganzen verunsichert.

Beide Briefe nutzen nur den Paratext der Anrede und des Abschlusses. Über das Datum wird der Leser nur durch den einbettenden Text informiert: Der erste Brief entsteht am 31. Mai 1591, der zweite am 3. Juni. Das erste Datum erfährt man nur aus dem Abschluss des Briefes, als Ereignis desselben Tages, an dem der Fürst, eine Palastbesetzung befürchtend, aus Alvinc nach Gyulafehérvár zurückfloh. Diese unglückliche Handlung verschlimmert die vergiftete Beziehung zwischen den beiden Báthorys noch weiter, und bewegt die Aristokratie von Siebenbürgen zur Entscheidung zwischen den zwei Söhnen. Der Brief entsteht und plädiert für die Versöhnung der Báthory-Söhne also genau an dem Morgen, an dem die Beziehung zwischen diesen sich endgültig verschlimmert. Diese Veränderung erfährt der Jesuit erst später, als er seine Pläne den neuen Informationen entsprechend gleich ändert und worüber er seinen Komplizen in einem weiteren Brief benachrichtigt.

Mehrere metanarrative Bemerkungen deuten auf die isochrone Erscheinung vorauf. Der Leser ist also in die simultanen Ereignisse eingeweiht, und dadurch darüber im Klaren, dass das Schreiben des Briefes so viel Zeit in Anspruch nimmt, dass dessen Inhalt von den simultanen oder darauf folgenden Ereignissen annulliert wird. ${ }^{37}$ Die Entstehungsdaten der Briefe umrahmen also den Wendepunkt in der historischen Binnengeschichte, die Sitzung des Senats am 1. Juni und dessen Urteil über Boldizsár Báthorys Untergang, worüber - nach wiederholter Verletzung der Schweigepflicht - der zweite Brief des Paters berichtet. Während der erste also die zukünftige Versöhnung der Gebrüder Báthory planend und sich auf Boldizsárs Aufstieg konzentrierend an Prolepsen reich ist, benutzt der zweite Brief vor allem Analepsen, da er über die Umstände der Entstehung der vorangehenden Entscheidung und deren Bekanntgabe berichtet. 
Trotzdem enthält auch der erste Brief retrospektive Teile. Cariglia wiederholt, ergänzt und begründet mehrere Paragraphen der früheren Sitzung und, um seinem Versprechen getreu den Grafen über den Stand ihrer Angelegenheit informieren zu können, über Ereignisse berichtet, die der Leser bis dahin aus einer anderen Perspektive gekannt hat. Durch die Vergegenwärtigungen hat der Leser angesichts der Möglichkeit der Technik der Selektion und Kombination die beiden Narrationen miteinander zu vergleichen.

Aufgrund des ersten Briefes halten wir Alfonso für einen guten Menschenkenner $^{38}$ und Diplomaten, da die Charakterisierung von beiden, Zsigmond und Boldizsár, mit den Früheren zusammenfällt, obwohl diese persönliche Perspektive gewisse Eigenschaften der kommunikativen Intention entsprechend speziell behandelt. Die zentrale Figur von Zsigmond wird in dem Roman stratoskopisch aus mehreren Perspektiven artikuliert, ${ }^{39}$ angefangen von István Báthorys Nachsinnen über Zsigmonds Porträt oder seine Charakterisierung von Kendi in dem Geheimrat, die in dramatischen oder erzählten Szenen weiter nuanciert werden. In seinem detailliert konstruierten Porträt malt der Erzähler ein komplexes Bild des Fürsten. Die Erzählzeit mit der erzählten Zeit konfrontierend zeigt er die Veränderung in der Interpretation und Beurteilung der historischen Figur und grenzt seinen erzählerischen Standpunkt betont und individuell von der allgemeinen Meinung ab. Aus der historischen Zukunft zurückblickend schildert er seinen geheimnisvollen, veränderlichen Charakter unabhängig von der aus der Geschichte bekannter Figur des Gewaltherrschers. ${ }^{40}$

Aus dieser komplexen Charakterzeichnung hebt Alfonso - wie er das mündlich vorher schon getan hat - in seinem Brief die Schwäche und Erschütterlichkeit des Fürsten hervor, ${ }^{41}$ durch welche Charakterzüge er als sein Beichtvater möglichst großen Enfluss auf seinen Günstling üben will. Die Beeinflussbarkeit des Fürsten ist in der früheren detaillierten erzählerischen Charakterzeichnung genauso betont, ${ }^{42}$ wie auch im zweiten Brief des Paters, außerdem wird es mit der Zeit zum entscheidenden Faktor des Handlungsfortgangs. Auch was die Figur Boldizsár Báthorys anbelangt, scheint der Briefschreiber eingeweiht zu sein, da er seine Jugend, seine Erziehung und seine Rolle in der nahen historischen Vergangenheit betreffend das Wissen des Lesers mit neuen Elementen erweitert, während er aufgrund der dem Leser schon bekannten Ereignisse ein - der Konklusion des Lesers ähnliches - treues Porträt des Grafen aus Fogaras zeichnet.

Alfonso - die Eitelkeit Boldizsárs analysierend - kommt zu der gleichen Konklusion ${ }^{43}$ wie der Graf in einer in dem Gedächtnis des Lesers noch gegenwärtigen Szene $^{44}$ über sich selbst. Weil das Ziel der Verbündeten der Aufstieg Boldizsárs und die Versöhnung der Gebrüder Báthory ist, wird der Ausgangspunkt des Báthory-Konfliktes, der Streit Boldizsárs mit Jósika, wachgerufen - eine Szene von Wichtigkeit nicht nur im Brief, sondern auch in dem ganzen Roman. Obwohl die Angelegenheit zwischen den Verbündeten bereits ins Gespräch gekommen 
ist, wird jetzt von dem fleißigen Briefschreiber über die Geschehnisse im Detail berichtet - unparteiisch, dem erzählerischen Diskurs getreu macht er aus den bekannten Ereignissen Auszüge. Er annotiert weiterhin die Verfestigung von Jósikas Position als Günstling, was auch gegen die Interessen der Prager ist, die die Versöhnung der Parteien und die Normalisierung der Situation in Transsylvanien vorläufig in dem Untergang des Günstlings garantiert sehen. Der Brief trifft noch keine Entscheidung in der früher mündlich schon diskutierten Frage, ob die hochstrebenden Pläne betreffend Jósikas oder Gyulais Absturz günstiger ist. Die Veränderungen entscheiden aber die Frage: Gyulai, der im Geheimrat mitgewirkt und über dessen Entschluss den Fürsten informiert hat, muss untergehen.

Der zweite Brief von Alfonso ${ }^{45}$ folgt bald dem ersten, obwohl der die Briefe einbettende Textteil nur sehr wortkarg über die Ereignisse dieser denkwürdigen Tage berichtet. Einerseits werden die Flucht nach Alvinc und die darauf folgende geheime Versammlung des Senats in Erinnerung gerufen, andererseits werden diejenigen Ereignisse bewusst verschwiegen, die den Jesuiten zum Schreiben des Briefes bewegt haben. Es wird also Ereignismosaiken geben, von denen der Leser nur durch persönliche Mitteilung erfährt.

Der zweiten Schrift geht folgende Einführung voran:

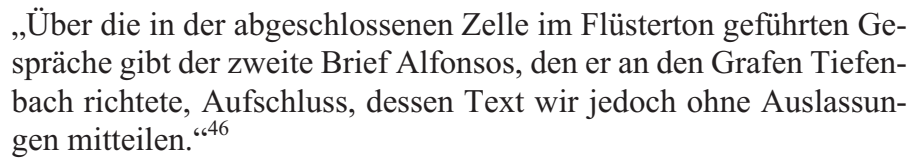

Der oppositionelle Konnektor der erzählerischen Zwischenrede wirft die Frage auf, mit welchem Textteil diese oppositionelle Beziehung bestehen vermag. Als Rückblende kann es sich auf den in der Zelle verschwiegenen Dialog beziehen, den der Leser bis dahin nicht einmal in Fragmenten kennt, also wird seine dadurch geweckte Neugier durch das letzte Drittel von Alfonsos weitschweifigem Brief erst viel später gestillt. Die Distanz zwischen Signifikaten und Signifikanten fällt durch diese lange Verzögerung auf. Syntaktisch ist diese Bemerkung auf einen früheren, bekannten Brief zwischen Alfonso und Tiefenbach rückführbar, als Anspielung auf die Fragmentiertheit des ersten Briefes.

Möchte man diese Leerstelle auf die erste Schrift beziehen, wird man enttäuscht, da keinerlei Spuren der Verkürzung zu finden sind, der erste Brief scheint auch nach dem Wiederlesen vollkommen und abgeschlossen zu sein.

$\mathrm{Zu}$ dieser Feststellung kommt man aber nur nach wiederholtem Lesen des Briefes vom 31. Mai. Unabhängig von der Interpretation zeigt die erzählerische Bemerkung, dass der Erzähler die einzelnen Abschnitte teils nur andeutend, teils 
aus verschiedenen Perspektiven schildernd, teils verschweigend oder in Details fast wörtlich wiedergebend dem Leser präsentiert - und sie so auf seine eigene Art und Weise gestaltet. Der Leser wird immer wieder an die Erfahrung der Textgestaltung erinnert, und der Erzähler erscheint in der Rolle des wohl informierten Narrators, der die Ereignisse vor der Veröffentlichung, sogar der Entstehung des Briefes zu kennen scheint.

Analeptische Allusion trifft der Leser an einer hervorgehobenen Stelle, wenn er über ein Ereignis erfährt, das sowohl die Ergänzung als auch den ganzen Roman betreffend von größter Wichtigkeit ist, nämlich die Geschehnisse und die Entscheidung der geheimen Sitzung des siebenbürgischen Senats. Cariglias Brief lernt der Leser aus der Perspektive des Schreibers kennen, er verfolgt den Text aus seiner Sicht. ${ }^{47}$ Der Brief berichtet über Ereignisse, denen er selber beigewohnt ist, wenigstens als Zuhörer, seine Position ist also im Vergleich zu den Geschichten ausschlaggebend, die Personen-, Raum- und Zeitverhältnisse werden im Verhältnis zu ihm interpretiert. Der Brief ruft mehrere Äußerungen von Anderen hervor, so entstehen mehrere ineinander verschachtelte erzählerische Zentren. Der Horizont der dadurch entstandenen erkennenden oder erzählerischen Ichs, ihr Zugang zu den Geschichten ist begrenzt und parteiisch, wodurch der Leser über die Geschehnisse aus verschiedenen Perspektiven mit unterschiedlichen Interpretationen erfährt. Bei der Darstellung dieser Äußerungen arbeitet der Schreiber mit verschiedenen Redeweisen. In seinen Part werden auf dramatische Weise Dialoge eingebaut, so wird die Rede der einzelnen Figuren lebendig. Denken wir nur an die Gergely zugeschriebene floskelhafte Einleitung des Briefes, deren Stil und Gleichnisartigkeit an frühere Äußerungen des Gesinnungslosen erinnern. Dieser umfangreiche Textteil kann auch als Verspätung betrachtet werden, die, weil Erzählzeit und erzählte Zeit zusammenfallen, die Spannung aller Zuhörer - Alfonsos, des ehemaligen Studenten, Tiefenbachs als des Angesprochenen und des Lesers - weiter steigt. Andere Textteile werden von dem sich gerade äußernden Subjekt syntaktisch getrennt, in freier indirekter Rede, mit den Bemerkungen, Eindrücken, Erklärungen des Schreibers ergänzt dargestellt.

Die Struktur der ineinander eingebauten narrativen Ebenen wird durch eine außergewöhnliche ${ }^{48}$ Fußnote oder in anderen Ausgaben durch eine eingeklammerte erzählerische Zwischenrede ${ }^{49}$ noch weiter kompliziert. In dieser Metalepse überschreitet der Erzähler die Rahmen seiner gerade erzählten Geschichte, spricht den Leser an, macht ihn aufmerksam darauf, dass das durch den Pater Geschriebene und Gesagte nicht zusammenfallen.

Die Metalepse $^{50}$ selbst erfüllt ihre Funktion, lässt das System der metadiegetisch ineinander gebauten fiktionellen Schichten nicht vergessen. Die konnotative Art des Ansprechens des Lesers motiviert zum Wiederlesen, weil man sich über die Existenz dieser Unterschiede nur dadurch Gewissheit verschaffen kann. ${ }^{51}$ 
Weil die Anrede des Lesers als Wegweiser gelesen wird, nimmt man an, dass die erwähnten Textteile wirklich existieren, sie existieren aber teilweise gar nicht oder nicht so, wie sie vom Narrator zitiert werden. Der Inhalt des Briefes und die Entscheidung des Geheimrates unterscheiden sich, gerade nicht an der Stelle, auf die der Erzähler die Aufmerksamkeit des Lesers lenkt, wesentlich voneinander. Zwischen Kendi und Gyulai gibt es keine Auseinandersetzung. Gyulai nimmt an und erfüllt ohne ein Klagewort die ihm auferlegte Aufgabe. ${ }^{52}$ Obwohl die Prämisse des Erzählers nicht stimmt, führt seine Schlussfolgerung zu einer äußerst interessanten Konklusion: Dadurch, dass er die Komplexität der narrativen Situation betont, spricht er den Pater von der Schuld der Lüge frei, da der Pater das von Zsigmond Gehörte weitergibt, und den durch die Entscheidung betroffenen Fürsten als primären Erzähler der Geschichte für die Verzerrungen verantwortlich macht. Der unbeständige Charakter des Fürsten zeigt sich auch in den - auch erzählten $^{53}$ - Veränderungen seiner Rezeption, wenn die Freude des ersten Hörens mit der Zeit zur Paranoia, die Dankbarkeit dem Boten gegenüber zum Ärger wird. Die Situation ist die wohl bekannte Situation des Missverständnisses in den Kemény-Romanen: ${ }^{54}$ Gyulai - als einziger seinem Eid getreu - darf nicht mehr sagen, der Fürst baut aus den Fragmenten einen paranoiden Geschichtsrahmen auf, in dem der Bote allmählich aus der Position des Günstlings in Ungnade fällt. Das zeigt sich darin, dass während der Fürst Gyulais aktiven Einsatz gegen Boldizsár in dem geheimen Rat erwartet, hält dieser nicht nur keine begeisternde Rede in dieser Angelegenheit, murmelt aber sogar seine hoffnungsvolle Worte nur vor sich hin. ${ }^{55}$ Trotz der komplizierten narrativen Situation wird aus der freien indirekten Rede klar, dass es sich hier um Zsigmonds Meinung handelt.

Die grenzüberschreitende Anrede des Lesers verletzt nicht nur die narrativen Rahmen, sondern hebt auch die komplizierte erzählerische Position hervor. Im Gegensatz zu dem allwissenden Erzähler der Einleitung wird der Leser hier mit der Ungenauigkeit der Kenntnisse des Erzählers, mit seinem mangelhaften Wissen konfrontiert. An dieser Stelle kennt der Narrator die Geschichte nicht gut oder führt den Leser bewusst irre, aber mit diesem Aufruf lenkt er die Aufmerksamkeit auf jeden Fall auf die falsche Wiedergabe der Inhalte, sich als Entwirren der Stränge schildernd, während er diese Stränge eigentlich noch weiter kompliziert.

Man darf aber einen weiteren wichtigen Faktor des Systems der Sprechsituationen nicht vergessen, nämlich dass die Veröffentlichung des Briefinhaltes nur durch den Bruch mehrerer Eide und die Verletzung mehrerer Schweigepflichte möglich ist. Gyulai ist der Einzige, der seinen Eid sowie sein Versprechen an den Senat und István Báthory hält, ${ }^{56}$ aber - wie man sehen wird - seine Treue zu dem gegebenen Wort führt zu seinem Untergang. ${ }^{57}$ Die in Cariglias Brief geschilderten Ängste des Fürsten rufen die ehemaligen Eide auf das Kreuz und das Schicksal der Verletzter dieser Eide wach, wodurch ein weiterer, bereits erzählter Teil evoziert wird. ${ }^{58}$ Aus dem dramatisch wortwörtlich hervorgerufenen Dialog zwischen 
dem Fürsten und Alfonso geht hervor, dass der Brief gerade den eigenen Entstehungsursprung erzählt, so, dass er mit verschiedenen Geschichten des Eidbruchs in einen historischen Kontext, eine Art Tradition des Eidbrechens eingebettet wird, wenn der Pater, um Zsigmond zu beruhigen, die unterschiedlichen Schicksale der Eidbrecher schildert. Wir haben also eine Geschichte der wiederholten Verletzung der Schweigepflicht - Gyulai, von der Schweigepflicht befreit, teilt dem Fürsten sein Geheimnis mit und nimmt dessen Eid ab, das Geheimnis zu bewahren. Der Fürst beichtet jedoch dem Abt, der die Schweigepflicht verletzend das Geheimnis mit Tiefenbach und Boldizsár verrät. Der Diskurs, der gerade gelesene Textteil offenbart sich dem Leser nur durch die Verletzung des Beichtgeheimnisses, durch den Einblick in das intime Geheimnis des Briefes. Dieses metadiegetisch auf verschiedenen Ebenen erzählte Motiv der Verschwiegenheit kann als selbstreflexive Konstruktion interpretiert werden, das ein analoges Verhältnis zwischen den Ebenen annehmend erlaubt, die eingebetteten Elemente, sogar die Narration als verkürzte Variante des Briefes (als mise en abyme) zu lesen.

Dieser Brieftext enthält als Intertext die Geschichte von Absolom aus dem zweiten Buch des Salomon aus dem Alten Testament, ${ }^{59}$ in das Zsigmond nach der Entscheidung des geheimen Rates, um sich beruhigen zu können, hineinblättert und anschließend über das Schicksal von Don Carlos nachdenkt. ${ }^{60}$ Die Parallele mit dem Alten Testament kommt nicht zum ersten Mal vor: In der Versammlung des Senats greift Kendi genau zu dieser Quelle bei der Charakterisierung der Báthory-Söhne. ${ }^{61}$ Boldizsár entspricht Absolom, Zsigmond entspricht Dávid, aber er beschäftigt sich mit der zwischen ihnen bestehenden Beziehung nicht, und die Lehre des Psalms soll sich nicht auf sie beziehen. Im Gegenteil dazu hebt Zsigmond aus der ersten Geschichte, aus dem Schicksal des Absolom, den inneren Kampf des gegen den Vater rebellierenden Sohnes, aus der zweiten des den rebellierenden Sohn auf das Schafott schickenden Vaters hervor.

Die Parallelen sind eindeutig, ${ }^{62}$ lassen den Leser trotzdem nachsinnen, weil sie statt des Kampfes zwischen Bruder und Cousin das Vater-Sohn-Verhältnis darstellen. In demselben Brief erwähnt Pater Alfonso zwar den gegen seinen Bruder jahrelang Krieg führenden und sein Land dadurch ins Chaos stoßenden Ludwig XI, ${ }^{63}$ aber er erwähnt ihn nur im Gespräch mit Tiefenbach als Gleichnis, Zsigmonds Parabeln gibt er keine weiteren Interpretationsmöglichkeiten.

Zsigmond sinnt in beiden Fällen über die Parabel von Vater und Sohn nach, im Sinne derer er Boldizsár als den gegen seinen Vater rebellierenden Sohn identifiziert, und daraus folgend sich selbst mit dem den Sohn zeugenden, ihn teilweise schaffenden Vater gleichstellt. Das Sohn-Sein ist dem Vater-Sein einerseits ähnlich, da er diese wiederholt, fortsetzt, vererbt, andererseits unterscheidet er sich in seinem Anderssein von ihm.

In dem Vater-Sohn-Verhältnis artikuliert sich die Wiederholung und die Fortsetzung. Die Macht wird aber von Vater zu Sohn weitervererbt, der Sohn folgt 
dem Vater nach dessen Tod oder durch seine (sakrale) Ermordung auf dem Thron, während der Bruder oder der Cousin nur in der Abwesenheit des leiblichen Nachwuchses oder nach dessen Tod an die Macht kommen kann.

Um seine Erregung gegen Boldizsár zu legitimieren, positioniert er sich nicht nur als Fürsten, sondern auch als Ursprung, als Vater über seinen Neffen, so dass ein angenommener Aufstand gegen ihn nicht nur die Verletzung der kollektiven Ordnung, sondern auch die Konfrontation mit der individuellen Abstammung bedeutet, was die Möglichkeit der Fortsetzung in Frage stellt. Durch den Sohnesmord wird sie aber unbedingt vernichtet.

Die Ähnlichkeit zwischen Zsigmond Báthory und seinem Vater, István, wird in der von Alfonso zitierten Traumszene deutlich ${ }^{64}$ wenn Zsigmond an der Grenze von Traum und Wirklichkeit die Ähnlichkeit zwischen dem königlichen Porträt an der Wand und Boldizsár bemerkt und sogar den anklagenden Blick seines Sohnes in Istváns Gesicht erkennt. ${ }^{65}$

Die Umdeutung der Rollen wird auch aus einer anderen Perspektive problematisiert, weil István Báthory seinen Günstling um Treue seinem Neffen, dem Woiwoden gegenüber bittet. Der Eid verliert aber mit dem Tod des polnischen Königs seine Gültigkeit, der Woiwode wird nämlich zum Fürsten, dem Gyulai, dem Text nach, keine Treue mehr schuldet. Seine inneren Kämpfe werden auch in seinem Tagebuch verewigt - aus dieser narrativen Situation kann der Leser die früheren Ereignisse aus der Perspektive der Titelfigur wiedererzählt kennen lernen. Gyulai sieht, dass Zsigmond seinem Vater nicht gewachsen ist und erfährt seine - bereits von István Báthory prognostizierte - Metamorphose und die Wendung seiner Taten ins Extreme, trotzdem kann er die Rolle des Kindes und des Fürsten nicht trennen, so garantiert er selbst die Kontinuität zwischen ihnen. Darauf, dass die veränderten Umstände seine Postion als Günstling umgedeutet haben, reflektiert er nicht. Wie er selber ahnt und vorausdeutet, hilft er Zsigmond durch die Tötung Boldizsárs seine Macht zu behalten, der aber ihn opfert und Siebenbürgen ruiniert.

Als Roman mit historischem Kern ist Gyulai Pál im Zusammenspiel von Geschichte und Literatur entstanden, das heißt, die wachgerufenen historischen Ereignisse und Personen wie auch ihre literarische Repräsentation erscheinen im Bewusstsein des Lesers simultan. Der historische Roman konstruiert seine Handlung aufgrund der als festgelegt betrachteten Ereignisse einer ausgewählten Epoche, zu deren authentischen Wiedergabe er sich jedoch nicht verpflichtet hat, kann also seiner fiktionellen Absicht nach von diesen Ereignissen abweichen. Infolgedessen wird der sich vor den Augen des Lesers entfaltende historische Roman als Konstruktion in zwei Diskurse eingebettet, die historischen und ästhetischen Horizonte sind im ständigen Zusammenspiel.

Einerseits sind die historischen Figuren, die bereits geschehenen und folgenden Ereignisse sowie ihr Ausgang gewissermaßen bekannt, ${ }^{66}$ andererseits werden 
den Tatsachen, die mit dem fiktionalen Mediumwechsel in einen neuen Kontext gesetzt werden, von den ursprünglichen unterschiedliche, neue Funktionen zugeteilt. Deshalb achtet man notwendigerweise darauf, wie sich seine geschichtlichen Vorkenntnisse, Vorurteile in einer künstlerisch geschaffenen Welt bestätigen.

Die Figur von Zsigmond Báthory ist also aus der kontinuierlichen, unveränderlichen historischen Person und aus der in dem Roman aus verschiedenen Perspektiven gezeigten Figur des jungen Woiwoden, später Fürsten und dem im Vergleich zur Erzählzeit zukünftigen Despoten zusammengesetzt. Das komplexe Charakterbild besteht also aus der historischen und der literarisch geschaffenen Figur, wobei die historische ebenfalls das Ergebnis einer historischen Erzählung ist. ${ }^{67}$ Dieser Zwiespalt bewegt den Leser dazu, bei dem Charakter des Fürsten zu verweilen, um die Kohärenz der Figur mit dem Anspruch auf ein textartiges Lesen zu schaffen. ${ }^{68}$

Zsigmonds Herrschaft bedeutet das Ende von Siebenbürgens Blütezeit. Abwechselnd verlässt und besteigt er den Thron mehrmals, was auch nicht dem Interesse des Landes dient. Seine unausgeglichene Politik führt zum Untergang des Fürstentums. Mit der Umgestaltung der Familienverhältnisse gibt Zsigmond eine Prognose seines eigenen Schicksals, nämlich dass sich die unter István Báthory herausgebildete Kontinuität des Fürstentums mit ihm auflöst. ${ }^{69}$

Cariglias zwei Briefe sind auch im Verhältnis zueinander von Interesse, besonders, weil die Zeilen des späteren Briefes die früheren immer wieder umschreiben. Indem nur Gyulais Schicksal erwähnt wird, bleibt in dem ersten Brief der Untergang der Günstlinge noch offen, aber die späteren Geschehnisse stabilisieren Jósikas Position Gyulai gegenüber. Gyulai ist kein aktiver Held, er ist durch Eid und Schweigepflicht gebunden, sein Schicksal ergibt sich aus den ihm zugeteilten Rollen. Er ist das jüngste Mitglied des geheimen Rates und als solcher hat er die Aufgabe, die Entscheidung des Rates dem Fürsten mitzuteilen - eine Rolle, die zu seinem Untergang führt, weil er dadurch den Zorn des Fürsten sowie von Boldizsár auf sich zieht. Statt eine historische Rolle zu spielen, wird er zum Mittel und Opfer von Verschwörungen.

Der Text erfüllt also einerseits die Erwartungen des Lesers: Nach einer Reihe von Komplikationen kommt der Brief - sein Ziel nie erreichend, aber mit einer Ergänzung erweitert - als Antwort an seinen Absender zurück. Die divergierenden Handlungsstränge treffen sich - der romantischen Handlungsführung entsprechend - zusammen. Andererseits spielt der Vergil ähnliche Erzähler mit seinem Leser in diesem romantischen Labyrinth und führt ihn absichtlich irre. So wie diese hypodiegetisch ineinander gebaute narrative Struktur, die Vervielfältigung der erzählerischen Rollen, die Unzuverlässigkeit der Erzähler und die Deformation der von ihnen gestalteten Geschichten und Nachrichten, fordert auch 
die selbstreflexive Konstruktion des mise en abyme eine aktive Anwesenheit des Lesers.

Diese zwei Verfahren funktionieren simultan und parallel. In dem romantischen Plotentwurf und der Realitätsnähe entdecken wir außertextuelle Elemente der Wirklichkeit, während im Erzählen und in der Konstruiertheit die modernen Merkmale der Textualität zur Geltung kommen. ${ }^{70}$

Anmerkungen

1 Szegedy-Maszák, Mihály: Kemény Zsigmond (Pozsony: Kalligram, 2007) (Erste Erscheinung: Budapest: Szépirodalmi, 1997).

2 Eine ähnliche Lesart präsentieren unter anderen folgende Studien: Benkő, Krisztián: Szinekdoché és önkivület. Apokalipszis-értelmezések Kemény Zsigmond A rajongók címü regényében. In: B. K.: Önkívület. Olvasónapló a magyar romantikáról (Pozsony: Kalligram, 2010) 192-239; Bényei, Péter: A történelem és a tragikum vonzásában: a történelmi regény müfaji változatai és a tragikum kérdései Kemény Zsigmond irásmüvészetében (Debrecen: Debreceni Egyetem Kossuth Egyetemi Kiadó, 2007); Eisemann, György: Elhallgatás, beszéd, szubjektum Kemény Zsigmond regényeiben. In: Iskolakultúra, 2007/1, 41-47; Gönczy, Mónika: Az Özvegy és leánya szövegvilágai. In: Studia Litteraria, Jg. 38. 2000, 84-113; Hites, Sándor: Prognózis és anakronizmus: A Zord idő mint politikai példázat. In: H. S.: A múltnak kútja. Tanulmányok a történelmi elbeszélések köréböl (Budapest: Ulpius-ház, 2004) 25-101; Kunkli, Enikő: A közösségi narratívák és egyéni önnarrativák összehangolásának esélyei Kemény Zsigmond Férj és nő cimü regényében. In: Studia Litteraria, Jg. 43. 2005, 115-143; Szegedy-Maszák, Mihály: Az újraolvasás kényszere (A rajongók) In: SZ-M. M.: Irodalmi kánonok (Debrecen: Csokonai, 1998) 72-93; Z. Kovács, Zoltán: Példázatosság és (romantikus) irónia Kemény Zsigmond három regényében (A szív örvényei, Férj és nő, A ködképek a kedély láthatárán) In: Z. K. Z.: ,„»Vanitatum Vanitas« Maga is a húmor “. Az irónia (korlátozásának) változatai a magyar romantika irodalmában (Budapest: Osiris, 2002) 139-186.

3 Zu der Romankomposition von Kemény und deren Rezeption siehe noch: Szegedy-Maszák, Mihály: Az elbeszélő nézőpont összetettsége Kemény Zsigmond regényeiben. In: MTA I. Osztályának Közleményei, 1979, 411-441, 428.

4 Kemény, Zsigmond: Eszmék a regény és a dráma körül. In: K. Zs.: Élet és Irodalom (Budapest: Szépirodalmi, 1971), 191-212, 208-209.

5 Martinkó, András: Töredékes gondolatok Kemény Zsigmond palackpostájáról 358-365. In: M. A.: Teremtö idök (Budapest: Szépirodalmi, 1977) 328-386, 368.

$6 \quad$ Szegedy-Maszák 2007, 86.

7 Kemény 1971, 200

8 Barta, János: Kemény Zsigmond írói világa. In: B. J.: Évfordulók (Budapest: Akadémiai, 1981) 235-267, 237.

9 Friedrich Schlegel: Levél a regényröl In: August Wilhelm Schlegel, Friedrich Schlegel: Válogatott esztétikai írások (Budapest: Gondolat, 1980) 370-382, 375.

10 Thomas Cooper: Zsigmond Kemény's Gyulai Pál: Novel as Subversion of Form. In: Hungarian Studies, 2002. Jg. 16/1, 29-50.

11 Paul Ricoeur: A történelem és a fikció keresztezödése (Üs. Jeney, Éva) In: P. R.: Válogatott irodalomelméleti tanulmányok (Budapest: Osiris, 1999) 353-372.

12 Kemény 1971, 196. 
13 Bényei 1999, 75.

14 Wolfgang Iser: Az olvasás aktusa. Az esztétikai hatás elmélete (Üs. Hárs, Endre) In: Kiss, Attila Atilla, Kovács, Sándor sk - Odorics, Ferenc (Hg.): Testes könyv I (Szeged: Ictus und JATE, 1996) 241-264. Vgl. Kemény, Zsigmond: Gyulai Pál II (Budapest: Szépirodalmi, 1967) 228.

15 Vgl. Szegedy-Maszák 1979, 411-441 und Szegedy-Maszák 2007, 89-97.

16 Kemény 1967, I. 259, 306.

17 Szegedy-Maszák, Mihály: A történet és az elbeszélés szereplői Kemény Zsigmond regényeiben. In: Irodalomtörténeti Közlemények, Jg. 1978/4, 475-494, 491.

18 Kemény 1967, II.128.

19 Kemény 1967, I. 360, 326.

20 Kemény 1967, II. 304.

21 Kemény 1967, I. 48.

22 Kemény 1967, I. 141.

23 Kemény 1967, I. 111.

24 Kemény 1967, I. 185.

25 Kemény 1967, I. 221.

26 Kemény 1967, I. 188.

27 Kemény 1967, II. 324.

28 Kemény 1967, I. 329.

29 Vgl. Cooper 2002, 30. Zu der Wichtigkeit der interpretativen Tätigkeit siehe noch Kemény, Zsigmond: Gyulai Pál (Hg.) Dobás, Kata (Budapest, Napkút, 2011) 282.

$30 \ldots$ mindig kikapcsolja a fószöveg terét és idejét [...] ott kezdődik, ahol az értelmező az addigi szinekdochikus vagy metonimikus előrehaladás megszakadását állapítja meg, és ott végződik, ahol ennek folytatását érzékeli." [... er schaltet Raum und Zeit des Haupttextes immer aus ... es beginnt dort, wo der Interpret den Abbruch des bis dahin synekdochischen oder metonymischen Fortgangs feststellt und endet, wo er die Fortsetzung dessen wahrnimmt.] [In meiner Übersetzung] Szegedy-Maszák, Mihály: Az irodalmi mü alaktani hatáselméletéröl. In: SZ-M. M.: Minta a szönyegen (Budapest: Balassi, 1995) 24-66, 39.

31 Den Begriff der Diegese benutzen wir in Anlehnung an Gérard Genette. In seiner Studie Diskurs der Erzählung unterscheidet er drei verschiedene Bedeutungsschichten der Erzählung. Die Erzählung in individuellem Sinne interpretiert er als Ergebnis des Prozesses der Textgestaltung, als narrative Aussage, d. h. als mündliche oder schriftliche Mitteilung bestimmter Ereignissequenz. Das Nacheinander der den Gegenstand des Diskurses bildenden wahren oder fiktiven Ereignisse nennt er Geschichten oder Historien, Narration nennt er den Prozess der Textgestaltung, den Akt des Erzählens der Geschichte. Bei der Aufstellung der erzählerischen Kategorien hält Genette auch den Gesichtspunkt vor Augen, ob der Narrator in der Geschichte als aktiv Mitwirkender, Teilnehmer, d. i. homodiegetisch, auftritt oder in einer heterodiegetischen Position des Außenseiters erscheint. Er untersucht, ob der Erzähler und der Akt des Erzählens Teil der Erzählung sind, er unterscheidet extradiegetische und intradiegetische erzählerische Situationen. Unter Ersterem versteht er die Narration ersten Grades, die Rahmenerzählung, wenn der heterodiegetische Erzähler die Geschichte einer Figur darstellt. Im Vergleich dazu ist die intradiegetische Situation von zweitem Grad, wo die erzählerische Tätigkeit einen Teil der Erzählung bildet, eine Figur der Erzählung zum Narrator wird und eine Geschichte erzählt. Die intradiegetische Narration ist also Narration innerhalb der Narration. Gérard Genette: Die Erzählung. (Hg.) Jochen Vogt (Üs. Andreas Knop) (München: Wilhelm Fink Verlag, 1998) 33-41, 48-53, 178.

32 Borisz Tomasevszkij: Irodalomelmélet In: A modern irodalomtudomány kialakulása, (Hg.) Bókay, Antal - Vilcsek, Béla (Budapest: Osiris, 2001) 268-286, 273. 
33 Kemény 1967, II. 26.

34 Kemény 1967, I. 248.

35 Kemény 1967, II. 162.

36 Die Figur der Gräfin Tiefenbach hat Kemény sehr beschäftigt, er plante sogar einen Roman mit diesem Titel, der Roman ist aber nie erschienen, ein Manuskript ist nicht bekannt. Siehe dazu den Brief von Kemény an Miklós Jósika vom 24. Februar 1847. Somogyi, Gréta, Pintér, Borbála (Hg.): Kemény Zsigmond levelezése (Budapest: Balassi - ELTE, 2007) 84. Vgl. Papp, Ferenc: Báró Kemény Zsigmond (Budapest 1922) Bd. I. 382.

37 Kemény 1967, I. 441-442.

38 Mit dem Fortgang des Textes müssen wir auch diese Feststellung unter Revision nehmen. Gergelys Charakterisierung von Alfonso kommt aus der Perspektive sowohl der Figur als auch des Erzählers falsch vor. Siehe: Cariglias Brief an Eleonora und Eleonoras Notizen in ihrem Tagebuch. Vgl. Kemény 1967, II. 273, 275.

39 Zu der unmittelbaren Charakterisirung Zsigmonds siehe noch: Szegedy-Maszák 2007, 106-107. Barta János rechnet Zsigmond zu den unberechenbaren, pathologischen Charakteren: Barta 1981, 244. Zu der Vielseitigkeit der Charaktere siehe noch Kemény 2011, II. 283.

40 Kemény 1967, I. 297-306.

41 Kemény 1967, I. 440.

42 An dieser Stelle hat man im Roman mit einer sehr komplizierten syntaktischen Situation zu tun. In dieser Charakterisierung kommt nämlich diese Eigenschaft als Frage vor, und die Antwort darauf entsteht nur nach langer Erläuterung. Vgl. Kemény 1967, 302, 305.

43 Kemény 1967, I. 407, 438.

44 Die äußerst geschickte Verwicklung der Stränge, die Verschachtelung der narrativen Ebenen zeigt auch die Tatsache, dass Boldizsár diese Aussage nach dem Lesen von Raelighs Brief macht, wonach er gerade von Alfonso aufgesucht wird, um ihn über das geheime Rat zu informieren, wie das im zweiten Brief des Paters erscheint.

45 Die Benutzung der Briefe und Tagebucheintragungen bezeichnenden Anführungszeichen ist inkonsequent. Sowohl hier als auch in der neuesten Auflage von 2011 fehlen die sekundären Mitteilungen bezeichnenden Anführungszeichen, vielleicht weil der Brief das Kapitel IV. des dritten Bandes abschließt. Vgl. Kemény, Zsigmond: Gyulai Pál (Pest: Hartleben, 1847). Bd. III. 106; Kemény 2011, 281.

46 Kemény 1967, I. 442 [in meiner Übersetzung].

47 Vgl. Szegedy-Maszák 1979, 423.

48 In den die Einführung oder den Abschluss der Briefe begleitenden Texten fügt der Erzähler den Äußerungen oft Kommentare hinzu oder unterbricht den Brief mit Zwischenreden der Figuren, aber von den in Keménys Romanen vorkommenden Briefen ist dieser der einzige, in den der Hinweis auf die Interpretation eingebaut ist.

49 In der von uns verwendeten Ausgabe von 1967 steht diese Ergänzung in Fußnote, während in der Erstausgabe und in der neu aufgelegten durchgesehenen Auflage von 2011 sie in Fußnote steht. Vgl. Kemény 1847, III. 95; Kemény 1967, I. 453-454; Kemény 2011, I. 275.

50 Auf das Phänomen der Grenzüberschreitung trifft man an mehreren Stellen, bei manchen Überschreitungen der Rahmen der verschiedenen narrativen Ebenen wird der Unterschied der Chronotopoi der Erzählung und der Geschichte besonders betont. Vgl. Kemény 1967, I. 185. Hier weist Kemény auf die zwischen 6.-17. Juli 1845 im Budapesti Híradó [Budapester Nachrichten] erschienenen Artikel von Emil Dessewffy hin, die er beim Schreiben des Roman Gyulai Pál gelesen hat. Für weitere Einzelheiten siehe den Brief an Miklós Jósika vom 15. Dezember 1845. Vgl. Somogyi, Pintér 2007, 59-60.

51 Kemény 1967, I. 453, I. 176, I. 179, I. 332.

52 Kemény 1967, I. 179. 
53 Kemény 1967, I. 454-455.

54 Die Ausgangssituation des Missverständnisses ist in mehreren Kemény-Romanen ausschlaggebend. Die Rollenwechsel in Ködképek a kedély láthatárán oder Özvegy és leánya erinnern an das Missverständnismuster der Shakespeare'schen Komödien, aber bei Kemény münden sie immer in Tragödien. Die Verwechslung der Rollen und die Fehlinterpretationen haben auch in Zord idö einen tragischen Ausgang, wie in A rajongók auch wortwörtliche Missverständnisse, also falsche Zeichendeutungen, zu dem Tod von Kassai Elemér führen. Siehe noch: Szegedy-Maszák 2007, 97, 105.

55 Vgl. Kemény 1967, I. 453, mit 176.

56 Schon als Farkas Kovasolczky Gyulai in einem Brief zur Versammlung des Senats einlädt, bittet er ihn in seiner kurzer Schrift, die Versammlung geheim zu halten.

57 Vgl. Németh G., Béla: Kemény Zsigmond. In: N. G. B.: Türelmetlen és késlekedö félszázad (Budapest: Szépirodalmi, 1971) 131-139, 135.

58 Vgl. Kemény 1967, I. 168, mit 451.

59 Absolom ist Davids Sohn, der sich als Erbe des Thrones gegen seinen Vater auflehnt. David flieht vor ihm aus Jerusalem. Absolom suchte seine Nebenfrauen auf, was ein Zeichen dafür ist, dass er die Macht übernahm. Vgl. 2 Sam 16, 21-22.

60 Die Kronologie nicht außer Acht lassend muss es sich hier um die historische Figur von Don Carlos eine bei weitem nicht sympathische Figur der Geschichte handeln. Er organisierte eine ungeschickte Konspiration gegen seinen Vater und wurde wegen Hochverrats und Konspiration für schuldig erklärt und zum Tode verurteilt. Don Carlos wird durch Schillers Drama aus dem Jahr 1787 und Verdis Oper zu einer positiven Figur gemacht.

61 Kemény 1967, I. 173.

62 Kemény 1967, I. 447.

63 Ludwig XI. kämpfte lange Zeit gegen seinen Bruder Karl den Kühnen, der gegen ihn sogar eine internationale Verschwörung organisierte, am Ende aber in der gegen Ludwig geführten Schlacht bei Nancy ums Leben kam. Seine Figur wird im Roman mehrmals erwähnt, zum Beispiel bei der Schilderung Zsigmonds Brutalität. Vgl. Kemény 1967, I. 298.

64 Kemény 1967, I. 455.

$65 \mathrm{Zu}$ der Wichtigkeit der Porträts siehe noch: Bence, Erika: Báthori Zsigmond arcképe: festmény és esszé Kemény Zsigmond Gyulai Pál címü regényében. In: Hid 2007. Jg. 5. 67-73.

66 Zsigmond Báthory überlässt Boldizsár den Thron für ein paar Tage, dann lässt er ihn ermorden und nimmt seine alte Position wieder ein. Zu der Bearbeitung der Geschichte von Zsigmond und Boldizsár Báthory siehe noch z. B. Márton, László: A nagyratörő (Budapest: Jelenkor, 1994), Passuth, László: Sárkányfog (Budapest: Szépirodalmi, 1960).

67 Siehe noch: Szegedy-Maszák, Mihály: Megforditott időrend. In: Sz-M. M.: Az újraolvasás kényszere (Pozsony: Kalligram, 2011) 220-225, 225.

68 Vgl. Szegedy-Maszák, Mihály: A Tündérkert müfaja és világképe. In: Sz-M. M.: A regény, amint írja önmagát (Budapest: Korona Nova, 1998) 57-79, 44.

69 Vgl. Hites, Sándor: Prognózis és anakronizmus: A Zord idő mint politikai példázat. In: H. S.: A múltnak kútja (Budapest: Ulpius-ház, 2004) 25-101, 58.

70 Viktor Žmegač, Történeti regénypoétika. A huszadik századi regény alapvető kettőssége (Üs. Rajsli, Emese) In: Thomka, Beáta (Hg.): Az irodalom elméletei I (Pécs: Jelenkor, 1996) 99-170, 104-115. 